Dye-sensitized solar cell with a pair of carbon-based electrodes

This content has been downloaded from IOPscience. Please scroll down to see the full text. 2012 J. Phys. D: Appl. Phys. 45165103

(http://iopscience.iop.org/0022-3727/45/16/165103)

View the table of contents for this issue, or go to the journal homepage for more

Download details:

IP Address: 139.179.14.46

This content was downloaded on 03/12/2013 at 09:43

Please note that terms and conditions apply. 


\title{
Dye-sensitized solar cell with a pair of carbon-based electrodes
}

\author{
Aung Ko Ko Kyaw ${ }^{1,3}$, Hosea Tantang ${ }^{2}$, Tao Wu ${ }^{3}$, Lin $\mathrm{Ke}^{4}$, Jun Wei ${ }^{5}$, \\ Hilmi Volkan Demir ${ }^{1,3,6}$, Qichun Zhang ${ }^{2}$ and Xiao Wei Sun ${ }^{1,7}$ \\ ${ }^{1}$ School of Electrical and Electronic Engineering, Nanyang Technological University, Singapore 639798, \\ Singapore \\ ${ }^{2}$ School of Material Science and Engineering, Nanyang Technological University, Singapore 639798, \\ Singapore \\ ${ }^{3}$ School of Physical and Mathematical Science, Nanyang Technological University, Singapore 639798, \\ Singapore \\ ${ }^{4}$ Institute of Material Research and Engineering (A*STAR), Singapore 117602, Singapore \\ ${ }^{5}$ Singapore Institute of Manufacturing Technology (A*STAR), Singapore 638075, Singapore \\ ${ }^{6}$ Department of Electrical and Electronics Engineering, Department of Physics and UNAM-National \\ Nanotechnology Research Center, Bilkent University, Bilkent, TR-06800, Turkey \\ 7 Tianjin Key Laboratory of Low-Dimensional Functional Material Physics and Fabrication Technology, \\ Tianjin University, 300072 Tianjin, People's Republic of China \\ E-mail: exwsun@ntu.edu.sg
}

Received 5 December 2011, in final form 26 February 2012

Published 2 April 2012

Online at stacks.iop.org/JPhysD/45/165103

\begin{abstract}
We have fabricated a dye-sensitized solar cell (DSSC) with a pair of carbon-based electrodes using a transparent, conductive carbon nanotubes (CNTs) film modified with ultra-thin titanium-sub-oxide $\left(\mathrm{TiO}_{x}\right)$ as the working electrode and a bilayer of conductive CNTs and carbon black as the counter electrode. Without $\mathrm{TiO}_{x}$ modification, the DSSC is almost nonfunctional whereas the power conversion efficiency (PCE) increases significantly when the working electrode is modified with $\mathrm{TiO}_{x}$. The performance of the cell could be further improved when the carbon black film was added on the counter electrode. The improved efficiency can be attributed to the inhibition of the mass recombination at the working electrode/electrolyte interface by $\mathrm{TiO}_{x}$ and the acceleration of the electron transfer kinetics at the counter electrode by carbon black. The DSSC with a pair of carbon-based electrodes gives the PCE of $1.37 \%$.
\end{abstract}

S Online supplementary data available from stacks.iop.org/JPhysD/45/165103/mmedia

(Some figures may appear in colour only in the online journal)

\section{Introduction}

Over the years, the efficiency of dye-sensitized solar cells (DSSCs) has been improved to more than $12 \%$, which is higher than that of amorphous silicon solar cells [1]. This level of power conversion efficiency (PCE) is sufficient to use DSSCs as an alternative renewable energy source in many applications if their cost could be further reduced. The high cost of DSSCs comes mostly from their electrodes, namely, platinum (Pt) or indium tin oxide (ITO) and fluorine-doped tin oxide (FTO). The working electrode is conventionally made of FTO, because of its high conductivity and transparency as well as its compatibility with the $\mathrm{TiO}_{2}$ sintering process at $450{ }^{\circ} \mathrm{C}$. Several approaches were attempted to replace FTO with cheap metal foils or substrates [2-5]. However, these approaches have a drawback in the reduction of photocurrent because the incident light must enter from the counter electrode. On the other hand, the counter electrode is made of FTO (or ITO) with a thin layer of $\mathrm{Pt}$ as a redox catalytic layer. This Pt catalytic layer is extremely important in DSSCs for the regeneration of the dyes after excitation. However, it is very expensive and corroded gradually by electrolytes $[6,7]$. In order to reduce the Pt-related negative factors, many studies have been carried out to find alternatives to replace the Pt catalytic layer. 
Recently, cobalt sulfide and poly (3,4-ethylenedioxythiophene) were used as a catalytic layer in DSSC with an organic redox couple [8]. Other popular materials are multiwalled carbon nanotube (CNT), single-walled CNT, carbon black, polyaniline and graphene [9-13]. In fact, carbon-based transparent electrodes as the replacement of conventional FTO/ITO have been widely used in several solid-state optoelectronic devices such as organic thin film transistors, organic light emitting diodes, organic solar cells and solid-state DSSCs [14-20]. However, to our surprise, there are fewer examples to consider replacing ITO (or FTO) together with the catalytic layer. Although Aitola et al reported that CNT-based films can be used as both conductive and catalytic layers, the fill factor of their DSSC is only $21.2 \%$ under one sun light due to poor catalytic activity [21]. In another report, a higher fill factor was achieved using vertically aligned N-doped CNT [22]. However, FTO is still required as the working electrode.

Therefore, it came to our attention that the dramatically reduced cost and the high stability of DSSCs could be achieved if FTO/ITO and Pt in DSSCs are replaced with carbon-based materials at both working and counter electrodes. In addition, carbon-based electrodes have other advantages over conventional FTO/ITO electrodes such as the abundance of material source, potentially scalable fabrication from the solution process, non-brittleness, chemical stability and optical transparency in the near infra-red region [16, 23-25]. However, in practice, DSSCs with a pair of carbon-based electrodes face two problems: (1) CNT and graphene exhibit very good conductivity but their catalytic activity is much poorer comparing with low-conductive carbon black for the counter electrodes and (2) the recombination is inevitable due to their catalytic activity to the redox reaction when carbon-based materials are used as the working electrodes. By addressing these two problems, we here report significant improvement in the replacement of all DSSCs' electrodes with carbon-based materials. In our FTO/ITO and Pt-free DSSCs, transparent conductive CNT films, which are modified with titanium-sub-oxide $\left(\mathrm{TiO}_{x}\right)$ for suppressing the charge transfer kinetics, are used as the working electrode, while the CNT films with thick carbon black layers are used as the counter electrode to increase both conductivity and catalytic activity, as illustrated in figure 1. We demonstrated that the characteristics of DSSCs improve considerably by modifying the CNT film with $\mathrm{TiO}_{x}$ and carbon black at the working and counter electrodes, respectively.

\section{Experimental details}

Pristine single-walled CNTs were purchased from Carbon Solution and used without further treatment. CNTs were dispersed in $0.5 \mathrm{wt} \%$ sodium dodecyl benzene sulfonate (SDBS) solution with the concentration of $0.2 \mathrm{mg} \mathrm{mL}^{-1}$ [26]. Probe sonicator (Sonics Vibra Cell) was used to make a homogeneous mixture. Its power and duration were set at $120 \mathrm{~W}$ (operating frequency $20 \mathrm{kHz}$ ) for $30 \mathrm{~min}$. The resulting solution was centrifuged at $6000 \mathrm{rcf}$ for $30 \mathrm{~min}$ and the supernatant was collected. The supernatant was directly airsprayed using air spray gun (Badger) onto glass substrates (a)
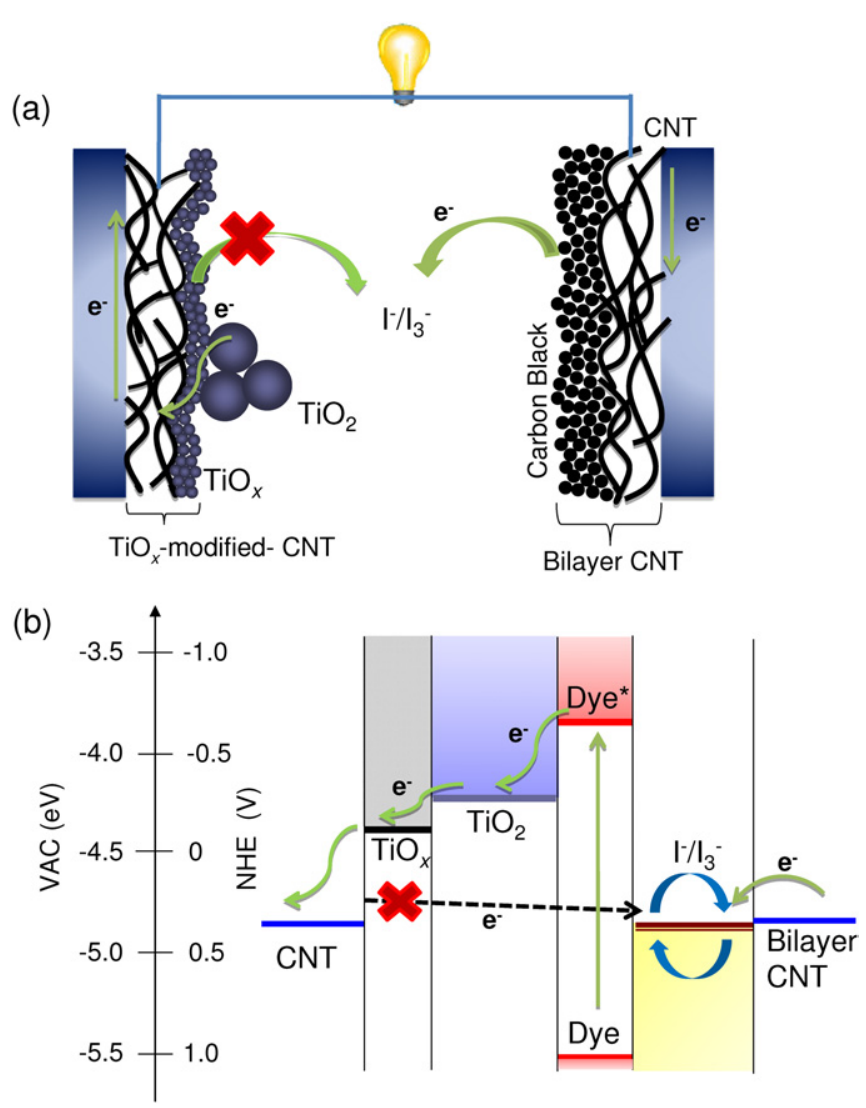

Figure 1. (a) Schematic of DSSC with a pair of carbon-based electrodes, where the recombination at the working electrode is inhibited by $\mathrm{TiO}_{x}$ layer while the charge transfer kinetics at the counter electrode is accelerated by carbon black and $(b)$ the energetic diagram of the individual components used in the cell

to deposit CNT films. During the spraying process, glass substrate was put on a hot plate with a temperature at around 80-90 ${ }^{\circ} \mathrm{C}$. The thickness of the CNT film was controlled by the spraying time. The thicknesses of the CNT film used for the working and counter electrodes are $80 \mathrm{~nm}$ and $250 \mathrm{~nm}$, respectively. After film deposition, the surfactant was removed by immersing in distilled water for $24 \mathrm{~h}$. Some CNT films are immersed in $98 \mathrm{wt} \%$ sulfuric acid for $30 \mathrm{~min}$ to yield p-doped CNT film. Carbon blacks used in the counter electrode were simply collected from a candle light.

$\mathrm{TiO}_{x}$ sol-gel was prepared by refluxing the mixture of $1 \mathrm{~mL}$ of titanium (IV) isopropoxide (Aldrich, 99.999\%), $5 \mathrm{~mL}$ of 2-methoxyethanol and $0.5 \mathrm{~mL}$ of ethanolamine in a threenecked flask under argon-gas environment at $80^{\circ} \mathrm{C}$ for $2 \mathrm{~h}$ and $120^{\circ} \mathrm{C}$ for $1 \mathrm{~h}$ [27]. The prepared sol-gel was spin-coated onto the CNT film at $3000 \mathrm{rpm}$ to make $\mathrm{TiO}_{x}$-modified-CNT electrode. The desired thickness of the film was controlled by further diluting the sol-gel with 2-methoxyethanol. Then, the samples were heated at $150{ }^{\circ} \mathrm{C}$ for $1 \mathrm{~h}$ in air. The precursor was hydrolyzed and converted to $\mathrm{TiO}_{x}$ during heating in air.

To fabricate DSSCs, the porous $\mathrm{TiO}_{2}$ film was first fabricated by screen-printing the $\mathrm{TiO}_{2}$ paste on CNT or $\mathrm{TiO}_{x}$-modified-CNT or commercial FTO (Nipon Sheet Glass, $15 \Omega / \square)$. The $\mathrm{TiO}_{2}$ film was sintered at $450{ }^{\circ} \mathrm{C}$ for half an hour in air. The thickness of the resulting $\mathrm{TiO}_{2}$ film is about $10 \mu \mathrm{m}$. After being cooled down to 
$80^{\circ} \mathrm{C}$, the photoanode was immersed in an ethanol solution containing $0.3 \mathrm{mM}$ cis-diisothiocyanato-bis(2,2'-bipyridyl4,'-dicarboxylato) ruthenium(II) bis(tetrabutylammonium) (Solarnonix, N719) overnight. Then, the sensitized photoanode was cleaned with ethanol to remove excess dye. A CNT or bilayer CNT or Pt-coated-FTO is used as the counter electrode. The two electrodes were bonded together by a $\sim 60 \mu \mathrm{m}$ thick thermal plastic. Finally, the electrolyte composed of $0.1 \mathrm{M} \mathrm{LiI}, 0.1 \mathrm{MI}_{2}, 0.5 \mathrm{M}$ terbutylpridine and $0.6 \mathrm{M}$ 3-hexyl-1-methylimidazolium iodide in methoxy-acetonnitrile was introduced between the electrodes by capillary action.

Photovoltaic measurement was conducted illuminating the devices under a solar simulator (Model 16S-002, Solar Light Company Inc.) with AM1.5G filter. The $J-V$ characteristics were performed using a Keithley 2400 sourcemeter. All devices were tested under ambient condition and simulated light intensity was adjusted to $100 \mathrm{~mW} \mathrm{~cm}^{-2}$ calibrated with an optical powermeter. Electrochemical impedance spectroscopy (EIS) measurements were carried out with Autolab system. $\mathrm{Ag} / \mathrm{AgCl}$ (KCl-saturated) and Pt wire were used as the reference and counter electrodes, respectively, in three-electrode system. The bias of $300 \mathrm{mV}$ was applied to avoid the mass-transport limitations due to diffusion in the electrolyte. The NOVA software was used to fit the impedance data for the charge-transfer resistance calculation. The sheet resistance was measured with a four-point-probe system while the thickness was measured by a surface profiler (KLA Tencor P-15). The optical transmittance spectra of the films were measured using the UV-vis-NIR spectrophotometer system (PerkinElmer Lambda 950) with an integrating sphere to capture directly transmitted light and forward scattered light. $\mathrm{X}$-ray diffraction (XRD) patterns of the films were obtained by a diffractometer (Siemens-model D5005) at $40 \mathrm{keV}$ and $36 \mathrm{~mA}$ using $\mathrm{Cu} \mathrm{K} \alpha(\lambda=1.54056 \AA)$.

\section{Results and discussion}

The scanning electron microscopy (SEM) image of a typical CNT film (produced by the air-gun spray method) is shown in figure 2(a). A number of well-entangled and interconnected CNTs with diameters in the range $10-25 \mathrm{~nm}$ were observed in the as-prepared film. The sheet resistance can be simply controlled by the thickness of the film but there is a tradeoff between its resistance and transparency. Figure 2(b) shows the relationship between the sheet resistance and the optical transmittance of the CNT films at optical wavelength of $550 \mathrm{~nm}$. The transmittance of the pristine CNT film (undoped) is as low as $55 \%$ at the sheet resistance of $500 \Omega / \square$. Around $80 \%$ transmittance, the sheet resistant of the pristine CNT film is more than $2000 \Omega / \square$. Clearly, such a film is not suitable for a transparent conductive electrode. Nonetheless, transmittance and sheet resistance of the CNT films can be optimized by doping with sulfuric acid. The acid treatment generates defects $\left(\mathrm{COOH}\right.$ and $\mathrm{SO}_{3} \mathrm{H}$ functionalities) on $\mathrm{CNT}$ and the produced chemical functionalities are beneficial for enhancing the electrical conductivity in the CNT film. The sulfuric acid p-dopes the CNT by oxidation and the attachment of chemical functionalities helps us to stabilize p-doping owing
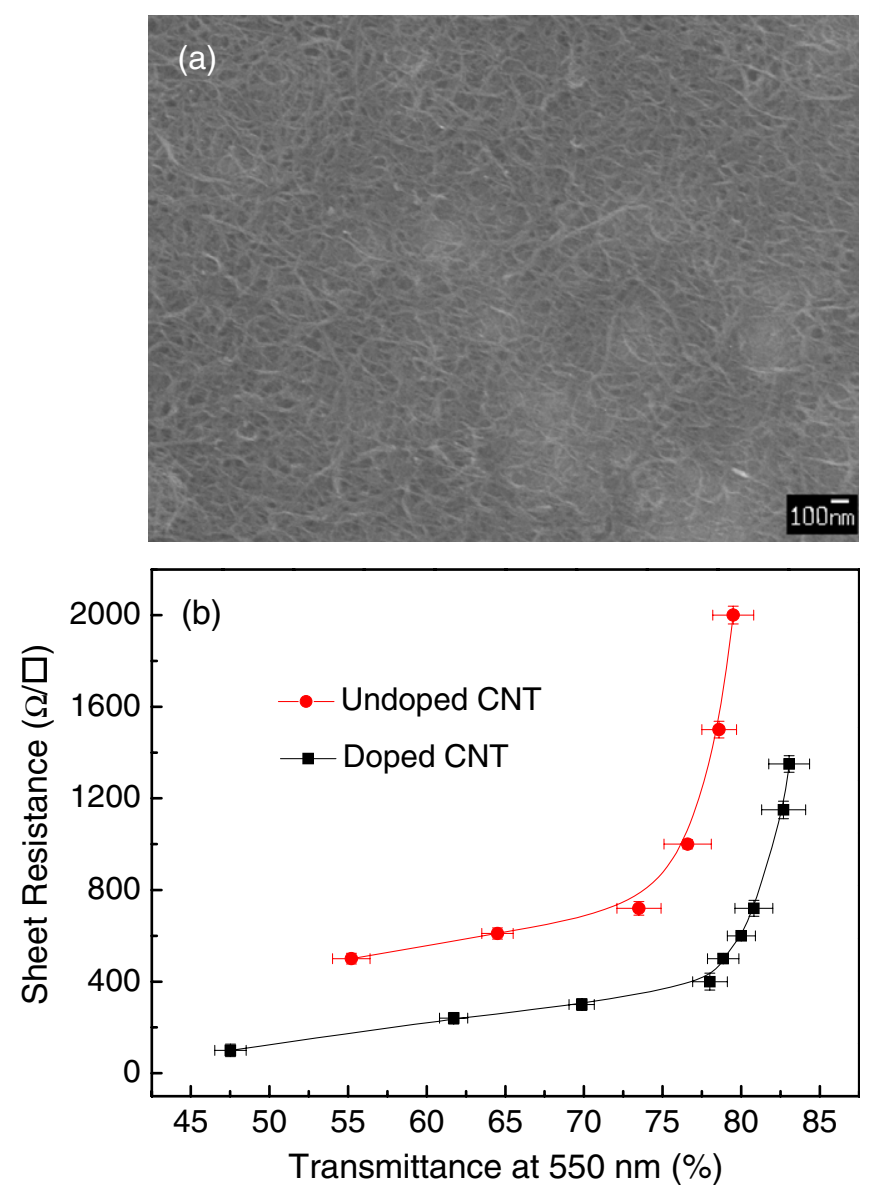

Figure 2. (a) SEM image of a typical CNT film deposited by air-gun spray. (b) The sheet resistance versus optical transmittance of the doped and undoped CNT films.

to their electron-deficient property [28, 29]. After doping, the transmittance of $\sim 80 \%$ is attainable at the sheet resistance of $\sim 500 \Omega / \square$. It is worth mentioning that the transmittance of the CNT film does not increase upon doping. However, as the sheet resistance is lower upon doping, the transmittance of the doped film becomes higher than that of undoped film when the films with the same sheet resistance are compared. The optimum combination of sheet resistance and transmittance occurs at $400-450 \Omega / \square$ and the corresponding transmittance is around $75-78 \%$. Hence, the doped-CNT films with a sheet resistance of $400 \pm 20 \Omega / \square$ were selected as the working electrodes. Since the optical transparency is not necessarily required for the counter electrode, the CNT film with a sheet resistance $90 \Omega / \square$ was used for the counter electrode to reduce the series resistance $\left(R_{\text {series }}\right)$ in the device despite its lower transparency.

Prior to the fabrication of DSSC with a pair of carbonbased electrodes, we separately made two DSSCs, each with a single carbon-based electrode. First, a DSSC with carbon-based counter electrode was fabricated using FTO as a working electrode and conductive CNT film $(90 \Omega / \square)$ as a counter electrode. Despite good conductivity, the conductive CNT film does not show strong catalytic activity, which was evaluated with charge-transfer resistance $\left(R_{\mathrm{CT}}\right)$ at the electrode/electrolyte interface, using EIS. $R_{\mathrm{CT}}$ was calculated by fitting the Nyquist plot of a three-electrode system in an 

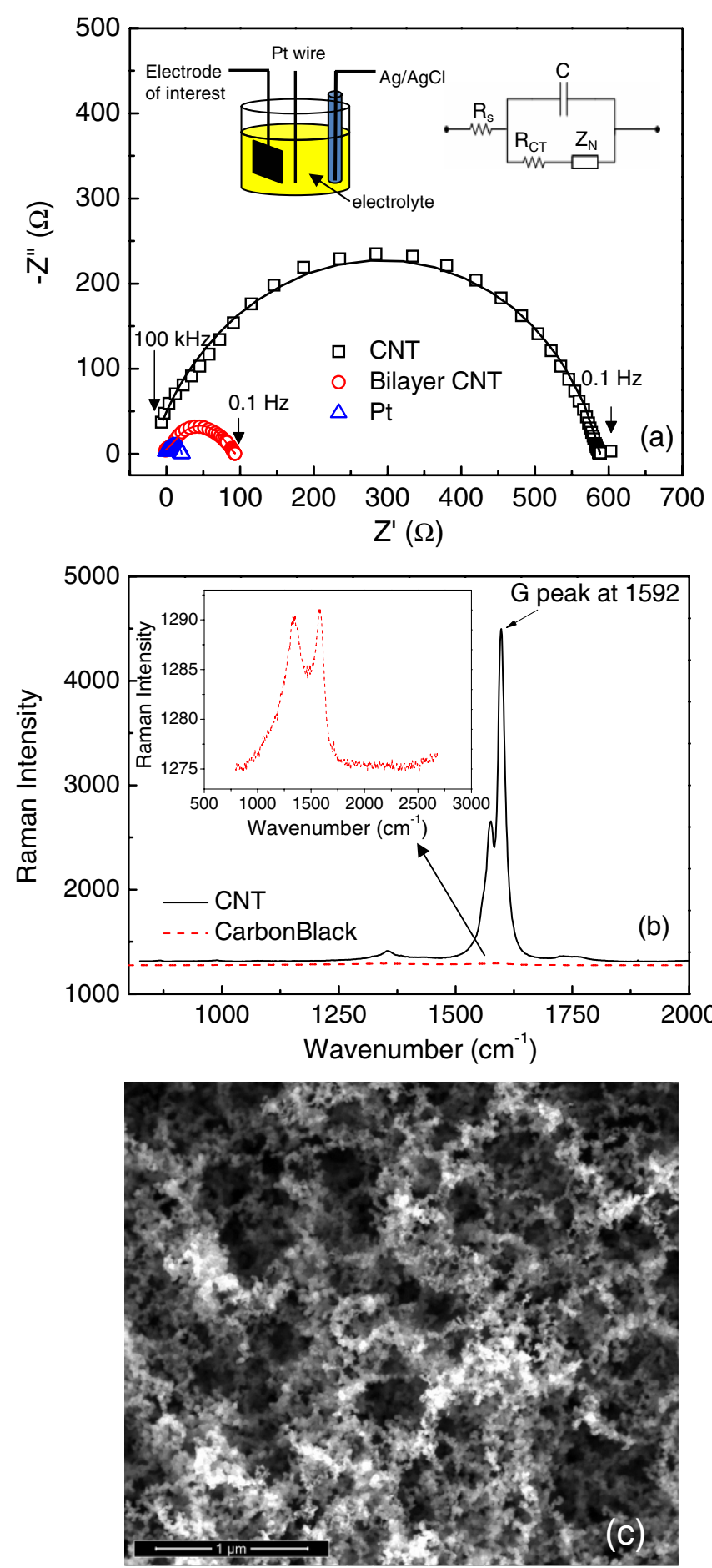

Figure 3. (a) The Nyquist plots of a three-electrode system with various electrodes of interest (CNT, Bilayer CNT and Pt). Inset (left) shows the experimental setup of three-electrode system. $\mathrm{Ag} / \mathrm{Ag}(\mathrm{Cl})$ and $\mathrm{Pt}$ wire were used as a reference and counter electrode respectively in the system. Inset (right) shows the equivalent circuit used in fitting data. Solid lines represent the fitted curves. ( $b$ ) The Raman spectroscopy of CNT and carbon black films. Inset is the detail spectroscopy of a typical carbon black film. (c) SEM image of a typical carbon black film.

electrolyte containing $0.05 \mathrm{M} \mathrm{I}_{2}$ and $0.5 \mathrm{M} \mathrm{LiI}$ in aqueous solution (figure $3(a)$ ). Herein, we used aqueous solution instead of non-aqueous solution such as acetonitrile because the former offered better reproducibility and stability [30]. The

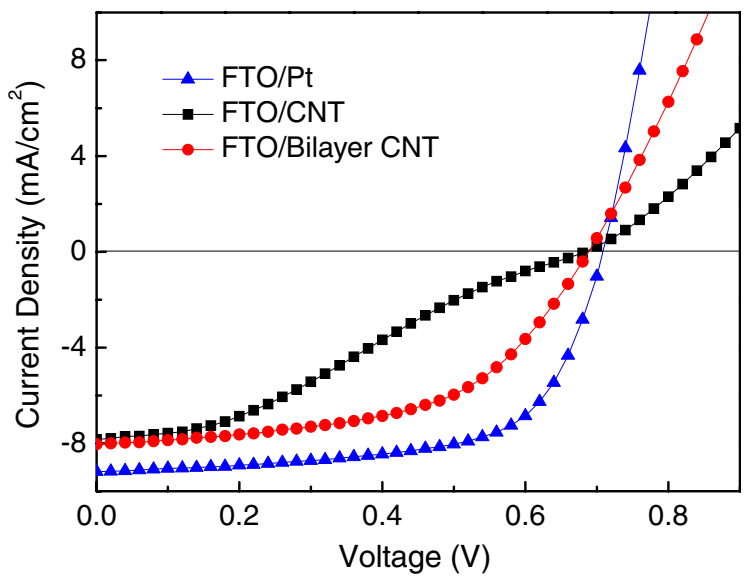

Figure 4. $J-V$ characteristics of DSSCs with carbon-based counter electrode and standard cell under simulated irradiation of AM1.5G (1 sun).

Nyquist plot was fitted with the equivalent circuit model (inset of figure $3(a)$ (right)). $\quad R_{\mathrm{CT}}$ indicates the electron-transfer resistance between the electrodes of interest and electrolyte containing redox species. The Nernst diffusion impedance $\left(Z_{\mathrm{N}}\right)$ describes the diffusion of $\mathrm{I}_{3}^{-}$in the electrolyte while $R_{\mathrm{s}}$ stems from the ohmic resistance of the electrolyte and the electrodes [31,32]. $R_{\mathrm{CT}}$ of the conductive CNT electrode is $311.4 \Omega \mathrm{cm}^{2}$, which is very large comparing with that of the Pt electrode $\left(15.57 \Omega \mathrm{cm}^{2}\right)$. It was reported that when CNTs are doped with electron-rich nitrogen, their catalytic activity increases due to the formation of metallic CNTs through band gap reduction [22,33]. We also found marginal improvement in the catalytic activity of nitrogen-doped-CNT electrode, whose $R_{\mathrm{CT}}$ is about $271.8 \Omega \mathrm{cm}^{2}$ (supplementary data, stacks.iop.org/JPhysD/45/165103/mmedia). A substantial increase in the catalytic activity was observed when thick carbon black $(10 \mu \mathrm{m})$ was loaded on top of the conductive CNT film (we shall call this combination as bilayer CNT). $R_{\mathrm{CT}}$ of the bilayer CNT electrode significantly reduces to $54.36 \Omega \mathrm{cm}^{2}$, which is comparable to that of Pt electrode. The Raman spectroscopy study showed that the as-prepared CNT films have a good quality with high $G / D$ ratio $(>20)$ (figure $3(b)$ ). On the other hand, carbon black films have low $G / D$ ratio, which might be due to the presence of defects in the carbon black. Because the active sites for catalysis in carbon materials are located at the crystal edges, carbon black with low crystallinity (defects) and many edges is more active than highly orientated CNT [11]. In addition, the thick layer with porous morphology may create additional active sites due to the large surface area (figure $3(c)$ ).

The current-voltage $(J-V)$ characteristics of DSSCs with carbon-based counter electrode and standard cell under illumination of AM1.5G simulated solar light $\left(100 \mathrm{~mW} \mathrm{~cm}^{-2}\right)$ are shown in figure 4. The electrical parameters of the fabricated DSSCs are listed in table 1. For simplicity, here we shall denote the name of DSSCs according to the electrodes used in the cell, for example, FTO/Pt cell stands for the DSSC with FTO working electrode and Pt counter electrode. The short-circuit current density $\left(J_{\mathrm{sc}}\right)$ and open circuit voltage $\left(V_{\mathrm{oc}}\right)$ of FTO/CNT and FTO/bilayer CNT are not much different 
Table 1. The electrical parameters of DSSCs with various electrodes.

\begin{tabular}{lllll}
\hline Cell (working electrode/counter electrode) & $J_{\mathrm{sc}}\left(\mathrm{mA} \mathrm{cm}^{-2}\right)$ & $V_{\mathrm{oc}}(\mathrm{V})$ & $\mathrm{FF}(\%)$ & $\mathrm{PCE}(\%)$ \\
\hline FTO/Pt & 9.186 & 0.708 & 65 & 4.22 \\
FTO/CNT & 7.835 & 0.684 & 30 & 1.63 \\
FTO/bilayer CNT & 8.037 & 0.688 & 54 & 2.98 \\
$\mathrm{CNT} / \mathrm{Pt}$ & 0.281 & 0.057 & 23 & 0.001 \\
$\mathrm{TiO}_{x}$-modified-CNT/Pt & 6.221 & 0.613 & 45 & 1.70 \\
$\mathrm{TiO}_{x}$-modified-CNT/CNT & 5.534 & 0.599 & 25 & 0.84 \\
$\mathrm{TiO}_{x}$-modified-CNT/bilayer CNT & 6.164 & 0.607 & 37 & 1.37 \\
\hline
\end{tabular}

while the fill factor increases substantially from $30 \%$ to $54 \%$ when the CNT film is replaced with the bilayer CNT film. The electron transfer kinetics between the bilayer CNTs and the electrolyte is fast enough to reduce triiodide $\left(\mathrm{I}_{3}^{-}\right)$ in electrolyte. This fast process prevents the undesirable recombination between the collected electrons at the working electrode and $\mathrm{I}_{3}^{-}$anions from the electrolyte and hence FF increases. The FF of FTO/bilayer CNT cell, however, is still lower than that of FTO/Pt cell $(65 \%)$. From the $J-V$ curve, the shunt resistances $\left(R_{\mathrm{sh}}\right)(\mathrm{d} V / \mathrm{d} I$ at $V=0)$ of FTO/bilayer CNT cell and FTO/Pt cell are similar but the $R_{\text {series }}(\mathrm{d} V / \mathrm{d} I$ at $I=0)$ of FTO/bilayer CNT cell is larger than that of FTO/Pt cell. This suggests that lower FF of FTO/bilayer CNT is mainly caused by a larger $R_{\text {series }}$ of the cell due to the larger sheet resistance of the bilayer CNT film compared with the Pt-coated-FTO film $(15 \Omega / \square)$, rather than the catalytic activity.

To use carbon-based materials as working electrodes in DSSCs, we faced a big challenge. Comparing with conventional FTO, although CNT and graphene have the similar work functions (FTO: $4.7-4.9 \mathrm{eV}, \mathrm{CNT}$ : $4.7-4.9 \mathrm{eV}$ and graphene: $\sim 4.6 \mathrm{eV}$ ), comparable optical transparency and similar electrical conductivity, no DSSC with carbonbased working electrode is successful. The reason is that the catalytic activity of carbon-based materials allows the recombination between the collected electrons and $\mathrm{I}_{3}^{-}$anions from the electrolyte. In order to solve this problem, we employed a facile method to modify the surface of carbonbased materials with an ultra-thin oxide layer, which can suppress the recombination between the collected electrons at the electrode and $\mathrm{I}_{3}^{-}$anions from the electrolyte [34]. Note that such modification should not affect the electrical and optical properties of the electrodes. Although this approach would solve the problem, the prerequisites for an ideal oxide used in the modification limits the choice of available materials. The possible reasons are (1) the oxide layer must convey electrons from the conduction band of $\mathrm{TiO}_{2}$ to the CNT electrode, i.e. the conduction band of this oxide must lie between the work function of CNT and the conduction band of $\mathrm{TiO}_{2}$; (2) the layer must be optically transparent within solar spectrum to prevent optical loss; (3) the oxide layer must be chemically stable especially in electrolyte and (4) the oxide layer must be compact enough to avoid the direct contact with electrolyte and CNTs. Luckily, these challenges can be solved through sol-gel process together with a low temperature annealing.

Herein, we modified the CNTs with sol-gel-processed $\mathrm{TiO}_{x}$, which was annealed at low temperature $\left(150^{\circ} \mathrm{C}\right)$, because the resulting materials satisfy all the criteria outlined above. In addition, $\mathrm{TiO}_{x}$ is able to inhibit the electron transfer between CNT electrode and redox containing electrolyte because of the poor catalytic property of metal which is contained in the metal oxide. It is well known that among over 75 metals in the periodic table, only the 12 in the group VIII and IB have strong catalytic properties. Other metals, including $\mathrm{Ti}$ in the group IV $\mathrm{B}$, are able to act catalytically but because of the great thermochemical difficulty of reducing them to the metallic state, and of keeping them reduced, they do not have strong catalytic property $[35,36]$. Such materials behave as inhibitor, rather than catalyst, in redox reaction.

The elemental composition of $\mathrm{Ti}$ and $\mathrm{O}$, cross-checked by $\mathrm{x}$-ray photoelectron spectroscopy, is $41.9 \%$ and $56.6 \%$, respectively (supplementary data, stacks.iop.org/JPhysD/45/ $165103 /$ mmedia). Since the ratio of $\mathrm{O}$ to $\mathrm{Ti}$ is between 1 and 2, the film is considered as titanium-sub-oxide, rather than titanium dioxide. As seen from the atomic force microscopy (AFM) image (figure 5(a)), the resulting $\mathrm{TiO}_{x}$ film is composed of myriad nano-sized colloids, which can cover the underlying CNT bundles almost completely. Because the heating temperature of the film is far below the crystallization temperature of the anatase or rutile phases of titanium oxide $\left(T_{\mathrm{c}} \geqslant 450{ }^{\circ} \mathrm{C}\right)$, the resulting film is amorphous as confirmed by XRD (figure $5(b)$ ). Despite the amorphous structure, the modification of CNTs with ultra-thin $\mathrm{TiO}_{x}$ layer slightly changes the overall sheet resistance of the electrodes. The sheet resistance of $\mathrm{TiO}_{x}$-modified-CNT film increases only to $550 \pm 20 \Omega / \square$ for $10 \mathrm{~nm}$ thick $\mathrm{TiO}_{x}$ from $400 \pm 20 \Omega / \square$ for CNT-only film. More interestingly, the $\mathrm{TiO}_{x}$ film is highly transparent in the visible region and near-infrared region, and hence, the optical transmittance of the $\mathrm{TiO}_{x}$-modified-CNT film is almost the same as that of the CNT film (figure $5(c)$ ). Despite an ultra-thin film, EIS test shows that $\mathrm{TiO}_{x}$ effectively inhibits the electron transfer kinetics at the CNT/electrolyte interface. $R_{\mathrm{CT}}$ of CNT electrode is only $311.4 \Omega \mathrm{cm}^{2}$ but that of $\mathrm{TiO}_{x}$-modified-CNT electrode increases to $2.513 \mathrm{k} \Omega \mathrm{cm}^{2}$ although it is smaller than that of FTO (figure $5(d)$, the experimental setup and equivalent circuit used for fitting are the same as that depicted in the inset of figure $3(a)$ ). Since $R_{\mathrm{CT}}$ varies inversely with the $\mathrm{I}_{3}^{-}$reduction activity of the electrode, the larger $R_{\mathrm{CT}}$ of the $\mathrm{TiO}_{x}$-modified-CNT film suggests that the reduction of $\mathrm{I}_{3}^{-}$at the CNT/electrolyte interface is strongly suppressed by the $\mathrm{TiO}_{x}$ layer.

$J-V$ characteristics of DSSCs with carbon-based working electrode under the illumination of AM1.5G simulated solar light $\left(100 \mathrm{~mW} \mathrm{~cm}^{-2}\right)$ are shown in figure $6(a)$. The PCE of $\mathrm{TiO}_{x}$-modified-CNT/Pt cell significantly improves to $1.7 \%$ with $J_{\mathrm{sc}}$ of $6.221 \mathrm{~mA} \mathrm{~cm}^{-2}, V_{\mathrm{oc}}$ of $0.613 \mathrm{~V}$ and FF of $45 \%$ while $\mathrm{CNT} / \mathrm{Pt}$ cell is almost nonfunctional. $J-V$ measurements 

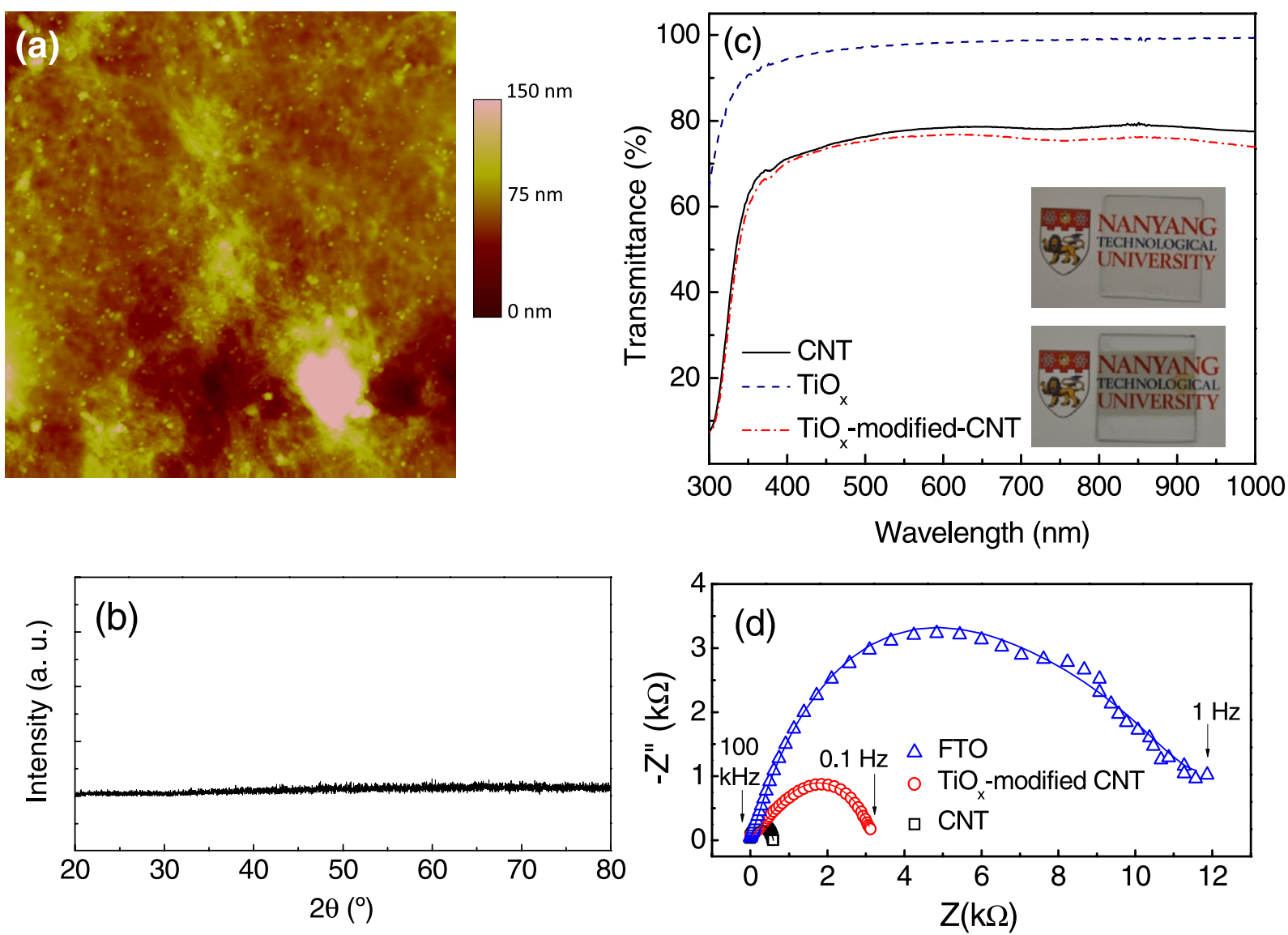

Figure 5. (a) AFM image of $\mathrm{TiO}_{x}$-modified-CNT film $\left(8.0 \times 8.0 \mu \mathrm{m}^{2}\right)$. (b) XRD spectrum of $\mathrm{TiO}_{x}$ film. $(c)$ Typical optical transmittance spectra of the CNT, $\mathrm{TiO}_{x}$ and $\mathrm{TiO}_{x}$-modified-CNT films. Inset: the photographs of $\mathrm{TiO}_{x}$ film (top) and $\mathrm{TiO}_{x}$-modified-CNT film (bottom) on the glass substrates. $(d)$ The Nyquist plots of a three-electrode system with various electrode of interest $\left(\mathrm{CNT} \mathrm{TiO}_{x}\right.$-modified-CNT and FTO). Solid lines represent the fitted curves.

of the cells in the dark also show that the leakage current (exhibiting a linear regime at negative voltages and low positive voltages) of $\mathrm{TiO}_{x}$-modified-CNT/Pt cell is much lower than that of CNT/Pt cell and even comparable to that of FTO/Pt cell (figure $6(b)$ ). The result clearly supports that the ultrathin $\mathrm{TiO}_{x}$ layer can efficiently stop the recombination between the collected electrons and $\mathrm{I}_{3}^{-}$anions from the electrolyte and can ensure the unidirectional flow of electrons in the cell. The $R_{\text {series }}$ of $\mathrm{TiO}_{x}$-modified-CNT/Pt cell, extracted from the $J-V$ curve under illumination, is $37.3 \Omega$ but that of FTO/Pt cell is only $9.4 \Omega$. The large $R_{\text {series }}$ of $\mathrm{TiO}_{x}$-modified-CNT/Pt cell can be attributed to the larger sheet resistance of the CNT film compared with that of FTO.

After verifying the feasibility of DSSCs with single carbon-based electrode, we fabricated DSSCs with a pair of carbon-based electrodes. The PCE of $\mathrm{TiO}_{x}$-modifiedCNT/CNT cell is $0.84 \%$ and could be improved to $1.37 \%$ in $\mathrm{TiO}_{x}$-modified-CNT/bilayer CNT cell thanks to the strong catalytic activity of bilayer CNT counter electrode (figure 7). Comparing with the $\mathrm{TiO}_{x}$-modified-CNT/Pt cell (with a single carbon-based electrode), the $\mathrm{TiO}_{x}$-modified$\mathrm{CNT} /$ bilayer CNT cell (with a pair of carbon-based electrodes) has the similar $J_{\mathrm{sc}}$ and $V_{\mathrm{oc}}$. Only the FF drops to $37 \%$ because the CNT film, which is less conductive compared with FTO, loads the additional series resistance on the cell. This can be verified by overlaying the $J-V$ curves of $\mathrm{TiO}_{x}$-modified-
CNT/Pt cell and $\mathrm{TiO}_{x}$-modified-CNT/bilayer CNT cell (not shown here).

Certainly, this is an initial attempt towards the direction of DSSCs with all carbon-based electrodes. Although the efficiency of the current cell with a pair of carbon-based electrodes is still somewhat lower than that of our own standard DSSC (the efficiency is $4.22 \%$ ), there is room to improve it. As mentioned above, low transmittance of the CNT film reduces the photocurrent, leading to a lower $J_{\mathrm{sc}}$. Moreover, we also observed the reduction in $V_{\mathrm{oc}}$ when FTO is replaced with the carbon-based working electrode. Generally, the $V_{\mathrm{oc}}$ of DSSC is logarithmically inversely proportional to the kinetic constant of the recombination of the collected electrons at the electrode with $\mathrm{I}_{3}^{-}$anions [31,37]. Therefore, some electrons are lost at CNTs/electrolyte interface although mass recombination is suppressed by $\mathrm{TiO}_{x}$. Low conductivity of CNT film also incurs additional series resistance in the cell and hence lowers FF. All these problems will be addressed in our following research and the enhanced performance is anticipated.

\section{Conclusions}

We demonstrated that carbon-based materials can be used as both working and counter electrodes in DSSC to eliminate expensive conventional electrodes completely. The conductive 

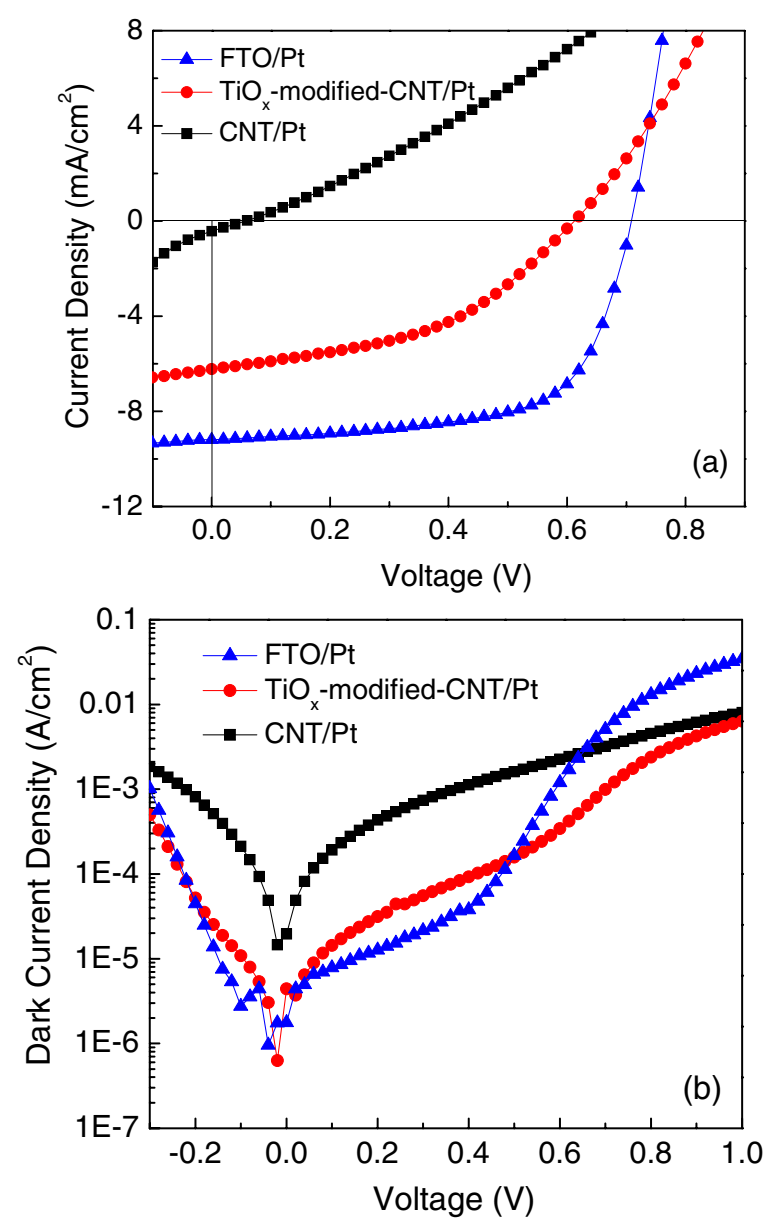

Figure 6. $J-V$ characteristics of DSSCs with carbon-based working electrode and standard cell $(a)$ under simulated irradiation of AM1.5G (1sun) and (b) in the dark in semilogarithmic scale.

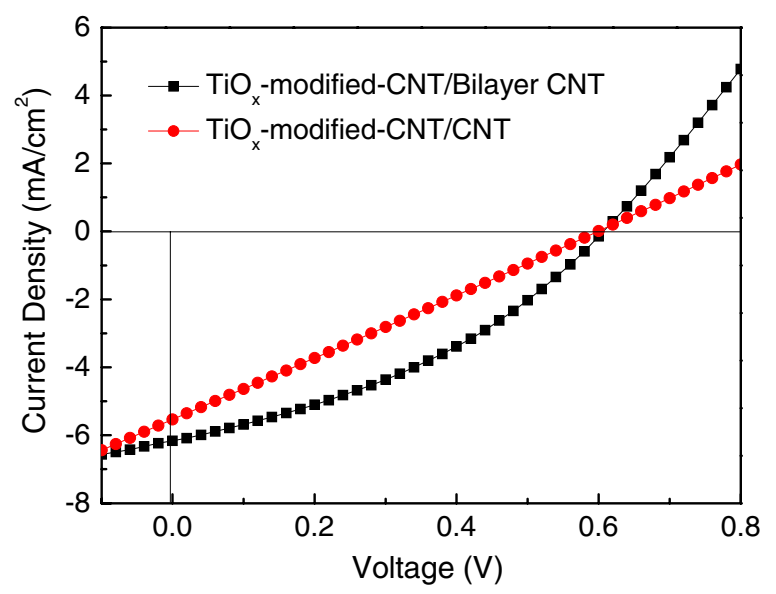

Figure 7. $J-V$ characteristics of DSSCs with a pair of carbon-based electrodes under simulated irradiation of AM1.5G (1sun).

CNT film is modified with an ultra-thin $\mathrm{TiO}_{x}$ layer at the working electrode to slow down the electron-transfer kinetics while thick carbon black is added on the conductive CNT film at the counter electrode to increase the catalytic activity. These implementations make DSSC with a pair of carbon-based electrodes feasible. To the best of our knowledge, this is the first demonstration of DSSCs with a pair of carbon-based electrodes. The efficiency is mainly limited by the low conductivity and transmittance of CNT films. With further optimization in the conductivity and transmittance of CNT films, DSSCs with all carbon electrodes can make the leap to emerge as a truly cost-effective and flexible DSSC.

\section{Acknowledgments}

This work was supported by the National Research Foundation (NRF-RF-2009-09, NRF-CRP-4-2008-04 and NRF-CRP-62010-02), New Initiative Fund (M58110108) and Start Up Fund (M5807000) from Nanyang Technological University, and National Natural Science Foundation of China (project numbers 61006037 and 61076015). Authors would like to thank Professor Hu Zheng from Nanjing University, China for providing nitrogen-doped CNT.

\section{References}

[1] Yella A, Lee H-W, Tsao H N, Yi C, Chandiran A K, Nazeeruddin M K, Diau E W-G, Yeh C-Y, Zakeeruddin S M and Grätzel M 2011 Science 334629

[2] Ito S, Ha N-L C, Rothenberger G, Liska P, Comte P, Zakeeruddin S M, Pechy P, Nazeeruddin M K and Gratzel M 2006 Chem. Commun. 4004

[3] Kyaw A K K, Sun X W, Zhao J L, Wang J X, Zhao D W, Wei X F, Liu X W, Demir H V and Wu T 2011 J. Phys. D: Appl. Phys. 44045102

[4] Onoda K, Ngamsinlapasathian S, Fujieda T and Yoshikawa S 2007 Sol. Energy Mater. Sol. Cells 911176

[5] Paulose M, Shankar K, Yoriya S, Prakasam H E, Varghese O K, Mor G K, Latempa T A, Fitzgerald A and Grimes C A 2006 J. Phys. Chem. B 11016179

[6] Olsen E, Hagen G and Eric Lindquist S 2000 Sol. Energy Mater. Sol. Cells 63267

[7] Luo Y, Li D and Meng Q 2009 Adv. Mater. 214647

[8] Burschka J, Brault V, Ahmad S, Breau L, Nazeeruddin M K, Marsan B, Zakeeruddin S M and Gratzel M 2012 Energy Environ. Sci. 56089

[9] Ramasamy E, Lee W J, Lee D Y and Song J S 2008 Electrochem. Commun. 101087

[10] Zhu H, Zeng H, Subramanian V, Masarapu C, Hung K-H and Wei B 2008 Nanotechnology 19465204

[11] Murakami T N et al 2006 J. Electrochem. Soc. 153 A2255

[12] Li Q, Wu J, Tang Q, Lan Z, Li P, Lin J and Fan L 2008 Electrochem. Commun. 101299

[13] Roy-Mayhew J D, Bozym D J, Punckt C and Aksay I A 2010 ACS Nano 46203

[14] Cao Q, Zhu Z-T, Lemaitre M G, Xia M-G, Shim M and Rogers J A 2006 Appl. Phys. Lett. 88113511

[15] Matyba P, Yamaguchi H, Eda G, Chhowalla M, Edman L and Robinson N D 2010 ACS Nano 4637

[16] Rowell M W, Topinka M A, McGehee M D, Prall H-J, Dennler G, Sariciftci N S, Hu L and Gruner G 2006 Appl. Phys. Lett. 88233506

[17] Wang X, Zhi L and Mullen K 2007 Nano Lett. 8323

[18] Wu J, Agrawal M, Becerril H c A, Bao Z, Liu Z, Chen Y and Peumans P 2009 ACS Nano 443

[19] Pasquier A D, Unalan H E, Kanwal A, Miller S and Chhowalla M 2005 Appl. Phys. Lett. 87203511

[20] van de Lagemaat J, Barnes T M, Rumbles G, Shaheen S E, Coutts T J, Weeks C, Levitsky I, Peltola J and Glatkowski P 2006 Appl. Phys. Lett. 88233503

[21] Aitola K, Kaskela A, Halme J, Ruiz V, Nasibulin A G, Kauppinen E I and Lund P D 2010 J. Electrochem. Soc. 157 B1831 
[22] Lee K S, Lee W J, Park N-G, Kim S O and Park J H 2011 Chem. Commun. 474264

[23] Dan B, Irvin G C and Pasquali M 2009 ACS Nano 3835

[24] Frackowiak E and Béguin F 2001 Carbon 39937

[25] Hu L, Hecht D S and Gruner G 2009 Appl. Phys. Lett. 94081103

[26] Geng H-Z, Kim K K, So K P, Lee Y S, Chang Y and Lee Y H 2007 J. Am. Chem. Soc. 1297758

[27] Lee K, Kim J Y, Park S H, Kim S H, Cho S and Heeger A J 2007 Adv. Mater. 192445

[28] Graupner R, Abraham J, Vencelova A, Seyller T, Hennrich F, Kappes M M, Hirsch A and Ley L 2003 Phys. Chem. Chem. Phys. 55472

[29] Tantang H, Ong J Y, Loh C L, Dong X, Chen P, Chen Y, Hu X, Tan L P and Li L-J 2009 Carbon 471867
[30] Trancik J E, Barton S C and Hone J 2008 Nano Lett. 8982

[31] Wang Q, Moser J-E and Grätzel M 2005 J. Phys. Chem. B 10914945

[32] Hauch A and Georg A 2001 Electrochim. Acta 463457

[33] Tantang H, Kyaw A K K, Zhao Y, Chan-Park M B, Tok A I Y, Hu Z, Li L-J, Sun X W and Zhang Q 2012 Chem.-Asian J. 7541

[34] Kyaw A K K, Tantang H, Wu T, Ke L, Peh C, Huang Z H, Zeng X T, Demir H V, Zhang Q and Sun X W 2011 Appl. Phys. Lett. 99021107

[35] Bond G C 1968 Platinum Met. Rev. 12100

[36] Clark A 1953 Ind. Eng. Chem. 451476

[37] Huang S Y, Schlichthorl G, Nozik A J, Gratzel M and Frank A J 1997 J. Phys. Chem. B 1012576 